Epilepsy

\section{Idiopathic generalised epilepsies: not only for the paediatrician}

\section{J W Sander}

\section{Adult onset IGE is more common than generally realised}

T he commonest epileptic syndrome is the so-called idiopathic generalised epilepsy (IGE), which accounts for at least a third of all cases of epilepsy in the community. ${ }^{1}$ This proportion is even higher in the paediatric age range. The syndrome is characterised by the presence of generalised tonic clonic, myoclonic, and typical absence seizures on their own or in different combinations. The onset is usually before the age of 16 . IGE has a typical electroencephalographic (EEG) pattern with paroxysms of generalised spike and wave and polyspike discharges, which is the hallmark of the syndrome. Age of onset and main seizure type are used to classify IGE further into four main subsyndromes: IGE with tonic-clonic seizures only, childhood absence epilepsy, juvenile absence epilepsy, and juvenile myoclonic epilepsy. As the majority of patients have the onset of IGE in childhood or adolescence, the international classification currently in use only recognises subsyndromes with onset in childhood Although it is well known in epilepsy specialist centres that IGE may present in adult life, this is often not well recognised by neurologists who do not have a specialist interest in epilepsy. Therefore, the paper by Marini et al (this issue, page 192-196) $)^{2}$ is of interest as they describe 34 consecutive patients with IGE beginning after the second decade of life, including several people over the age of 50 presenting with "de novo" IGE. The authors quite rightly suggest that adult onset IGE is more common than generally realised and this is an important takeaway message. The second message is the importance of a directed diagnostic EEG strategy in all patients presenting with a first unprovoked epileptic seizure independent of age. This goes against the suggestion that after the age of 25 years, EEG is less important as an investigative tool in epilepsy. ${ }^{3}$ The authors' practice is to record an early postictal EEG, followed by a sleep deprivation EEG in negative cases and they have previously shown that this leads to a higher diagnostic yield in first seizure cases in all ages. ${ }^{4} \mathrm{~A}$ second spin off seems to be a relatively frequent identification of adult-onset IGE that might otherwise have been regarded as being partial epilepsy of uncertain origin. The paper also highlights the growing importance of genetics in epilepsy, particularly in IGE. ${ }^{5}$ Early onset IGE has a genetic aetiology with complex inheritance. Family and twin studies suggest a common genetic origin for all subtypes of IGE and this seems also to apply to the late onset form. Currently, intensive work is ongoing to clarify fully the genetic blueprints of IGE, and the Melbourne group, which authored this article, are major players in this area. For the general neurologist the recognition of adult-onset IGE has implications for accurate diagnosis and treatment. Adults with a family history of IGE and in whom other causes have been ruled out are prime candidates for adultonset IGE. The diagnosis of adult-onset IGE can avoid unnecessary investigations, direct appropriate treatment, and allow an optimistic prognosis, and this should not be forgotten.

J Neurol Neurosurg Psychiatry 2003;74:147

\section{Author's affiliation}

J W Sander, Department of Clinical and Experimental Epilepsy, UCL Institute of Neurology, National Hospital for Neurology and Neurosurgery, Queen Square, London WCIN 3BG, UK

Correspondence to: J W Sander;

Isander@ion.ucl.ac.uk

\section{REFERENCES}

1 Sander JW, Shorvon SD. Epidemiology of the epilepsies. I Neurol Neurosurg Psychiatry 1996;61;433-43.

2 Marini C, King MA, Archer JS, et al. Idiopathic generalised epilepsy of adult onset: clinical syndromes and genetics. J Neurol Neurosurg Psychiatry 2003;74: 192-6.

3 SIGN. Diagnosis and Management of epilepsy in adults. Scottish Intercollegiate Guidelines Network. Edinburgh: Royal College of Physicians, 1997

4 King MA, Newton MR, Jackson GD, et al. Epileptology of the first-seizure presentation: a clinical, electroencephalographic and magentic ressonance imaging study. Lancet 1998;352:1007-11.

5 Johnson MR, Sander JW. The clinical impact of epilepsy genetics. J Neurol Neurosurg Psychiatry 2001;70:428-30.

\title{
Dipoles and dementia
}

\section{M Epstein}

Can the link between MEG dipoles and cortical activity lead to increased applications for the unique capabilities of MEG?

$F$ or almost two decades magnetoencephalography (MEG) has been the stepchild of functional neuroimaging. Despite its evolution from single sensors to arrays of more than 100, with unmatched temporal resolution, the legitimacy of MEG as a clinical and research tool has been repeatedly challenged. On the one hand, neurophysiologists persistently-and appropriatelyquestion the advantage of MEG over scalp-recorded electroencephalograms and evoked potentials when the latter are analysed by sophisticated algo- rithms. On the other hand, advocates of functional magnetic resonance imaging (MRI) and positron emission tomography have disparaged MEG because of the assumptions required to obtain unique solutions from magnetic field data. The paper by Maestú et al (this issue, page 208-212) ${ }^{1}$ provides an example of work that is establishing the capabilities of MEG.

Maestú et al used MEG to compare patients with Alzheimer's disease and elderly subjects. They had previously found that reduced MEG activation was present in left temporal and parietal areas during a probe letter working memory task; this reduction correlated with results from neuropsychological assessments and activities of daily living. In the present study, they report that 
magnetic field measures correlate significantly with the relative volume of lateral and mesial temporal regions. Consistent with the long latency of cognitive evoked potentials, the MEG features of interest occurred more than 400 ms after stimulus onset. After a Bonferroni correction, the only correlation coefficient considered significant was that between left temporal lobe activity and the relative volume of the left hippocampus. The smaller the number of late MEG activity sources in the left temporal region, the greater the atrophy in the mesial portion of the left temporal lobe. Definable changes in MEG may correspond to the exquisite anatomic resolution of MRI.

Different types of algorithms, embodying different assumptions, have been employed to solve for the fourdimensional origin of electrical activity within the brain. The commonest algorithm models magnetic flux as the product of single equivalent current dipoles.
Despite its simplicity, this approach has given good results when compared with invasive recordings. A straightforward MEG technique is to plow mathematically through a second or so of evoked potentials, solving for dipoles every $4 \mathrm{~ms}$ and identifying those that show high goodness of fit and a restricted intra cranial volume. Analysis of a single evoked potential can produce hundreds of dipoles scattered across multiple brain regions. This approach provides interesting results, and the assumptions behind it seem reasonable: but it's fair to ask whether all those dipoles represent something "real", or are merely artifacts of elaborate computer processing.

The report of Maestú and colleagues suggests that the plethora of dipoles is a valid reflection of brain activity. Significantly fewer of them are found in the atrophic left temporal lobes of Alzheimer's disease patients compared to elderly subjects. Although we still don't know what the individual dipoles mean, we have increasing reason to believe that they do reflect neuronal processing. We can hope that a greater understanding of the link between MEG dipoles and cortical activity will lead to increased applications for the unique capabilities of MEG.

J Neurol Neurosurg Psychiatry 2003;74:147-148

\section{Author's affiliation}

C M Epstein, Emory Clinic, Department of Neurology, 1365 Clifton Road, Atlanta, GA 30322, USA

Correspondence to: $\operatorname{Dr} C M$ Epstein; chipstein@aol.com

\section{REFERENCE}

1 Maestú F, Arrazola J, Fernández A, et al. Do cognitive patterns of brain magnetic activity correlate with hippocampal atrophy in Alzheimer's disease? J Neurol Neurosurg Psychiatry 2003;74:208-12.

\section{Multidisciplinary rehabilitation for people with Parkinson's disease}

\section{E D Playford}

\section{Worse general and mental health for patients receiving rehabilitation?}

There is little evidence available to support or explain the role of rehabilitation in progressive neurological disorders, so the paper by Wade et al (this issue, page 158-162) is a welcome contribution. ${ }^{1}$ The paper examines whether patients with Parkinson's disease have sustained benefit following a multidisciplinary outpatient rehabilitation programme. The programme comprised individualised multidisciplinary interventions and a group education programme and was delivered to six patients at a time over six weeks. The findings suggest that six months after randomisation those receiving rehabilitation had worse general and mental health and that their carers had a trend to more strain. This contrasts with findings from studies of the rehabilitation of people with multiple sclerosis ${ }^{2}$ and brain injury. ${ }^{3}$ Why should this conscientiously performed intervention have apparently negative effects?

The authors themselves suggest a number of reasons, including previously satisfactory management leaving little room for improvement, limited input, little psychological or medical input, need for continued input, and finally, the use of inappropriate measures. These reasons may all provide an explanation for the lack of benefit but do not explain the negative impact. To explain this we should consider further the nature of the intervention and it's assessment.

In this study the multidisciplinary therapy input was assessed using two main measures. The impact of the physiotherapy intervention was measured using a sit-stand-walk time and showed a trend towards improvement. The occupational therapy components were not measured directly and the speech and language therapy aspects were measured on a 5 point scale, which may be relatively unresponsive.

The authors also measured health related quality of life. Interestingly this deteriorated in the intervention group. Health related quality of life (HRQoL) may be defined as the patients' appraisal of their current level of functioning and their satisfaction with it compared with what they perceive to be ideal. In the context of this study it will be influenced by the whole intervention, including the educative aspects of the programme. This will include the day to day education provided by the therapists as part of the treatment sessions, as well as the formal education programme. There is now considerable expertise in the areas of patient education. Teaching packages should enhance self-management and self efficacy. ${ }^{4}$ The patients recruited to this study are not described in terms of disease severity and duration but patients early after diagnosis will have very different needs to those who are entering a more complex stage of the disease with an unpredictable response to medication. Mistimed education programmes may be seen as trivial or intrusive and distressing. There is evidence that significant parts of the variance of HRQoL are accounted for by mood ${ }^{5}$ but, as pointed out by the authors, doctors and psychologists were not involved in this study.

This is a useful contribution to the literature but the findings emphasise the need for clarity when planning interventions and the measurement of their benefit. The design of rehabilitation trials is a developing science, but two questions need to be kept in mind. Firstly, does the intervention work and, secondly, is the intervention of value to the patient? In this study the intervention may have worked in some areas but not others, but despite this the patients still appeared to value it. Clarifying the 
reasons for these findings is important so the design of future trials can be improved.

J Neurol Neurosurg Psychiatry
2003;74:148-149

\section{Author's affiliation}

E D Playford, Institute of Neurology, Queen Square, London WC1N 3BG

\section{REFERENCES}

1 Wade DT, Gage H, Owen C, et al. Multidisciplinary rehabilitation for people with Parkinson's disease: a randomised controlled study J Neurol Neurosurg Psychiatry 2003;74: 158-62

2 Freeman JA, Langdon DW, Hobart JC, et al. Inpatient rehabilitation in multiple sclerosis: do the benefits carry over into the community? Neurology 1999;52:50-6.
3 Eames P, Cotterill G, Kneale TA, et al. Outcome of intensive rehabilitation after severe brain injury: a long-term follow-up study. Brain Inj 1996;10:631-50.

4 Bodenheimer T, Lorig K, Holman $\mathrm{H}$, et al. Patient self-management of chronic disease in primary care. JAMA 2002;288:2469-75.

5 Schrag A, Jahanshahi M, Quinn N. What contributes to quality of life in patients with Parkinson's disease? J Neurol Neurosurg Psychiatry 2000;69,308-12

Correspondence to: E D Playford;

d.playford@ion.ucl.ac.uk

\section{NEUROLOGICAL PICTURE}

\section{Vasospastic amaurosis fugax}

A

54 year old man was admitted because of repeating (10-12/day) visual disturbances in the left eye. He reported shrinkage of the visual fields and a shadow-like visual impairment progressing to complete darkness within about $3 \mathrm{~min}$ and lasting about $10 \mathrm{~min}$, followed by complete recovery. There was no personal history of hypertension, diabetes, and smoking. General medical examination was normal. The blood pressure was $130 / 80 \mathrm{~mm} \mathrm{Hg}$. Investigations of standard haematological and biochemical parameters revealed elevated low density lipoprotein cholesterol $4.45 \mathrm{mmol} / \mathrm{L}$ (normal $<3.35 \mathrm{mmol} / \mathrm{L}$ ) and elevated triglycerid level $2.67 \mathrm{mmol} / \mathrm{L}$ (normal range $0.45-1.80 \mathrm{mmol} / \mathrm{L}$ ). Ultrasound studies of the extracranial vessel showed normal intima-media thickness $(0.9 \mathrm{~mm})$ and no plaque formation in the carotid bulb. Complete cardiological examination, including electrocardiography, transthoracic echocardiography, and chest $x$ rays, were unremarkable. Magnetic resonance imaging of the brain was unremarkable, as was conventional angiography.

During examination of the retinal circulation by laser Doppler by scanning laser Doppler flowmetry ${ }^{1}$ elaborating a map of perfused retinal vessels and capillaries, the patient suffered a visual disturbance as described above. The retinal perfusion map (fig lA) revealed impaired perfusion of the retinal temporal artery at the 11 and 12 o'clock positions (arrows), which resolved within 21 min (Fig 1B). A treatment with statin to lower lipids and the antiplatelet agent copidogrel was initiated, but without influence on the events. Suspecting vasospastic amaurosis fugax, ${ }^{2-4}$ we supplemented the therapy by calcium-channel blocker cyclandelate (1200 mg daily), ${ }^{2}$ which yielded significant improvement of the clinical condition.

\section{References}

1 Michelson G, Schmauss B, Langhans M, et al. Principle, validity, and reliability of Scanning Laser Doppler Flowmetry. J Glaucoma 1996;5:99-105.

2 Winterkorn J, Kupersmith M, Wirtschafter D, et al. Treatment of vasospastic amaurosis fugax with calcium-channel blockers. New Engl J Med 1993;329:396-8.

3 Bernard GA, Benett JL. Vasospastic amaurosis fugax. Arch Ophthalmol 1999;117:1568-9.
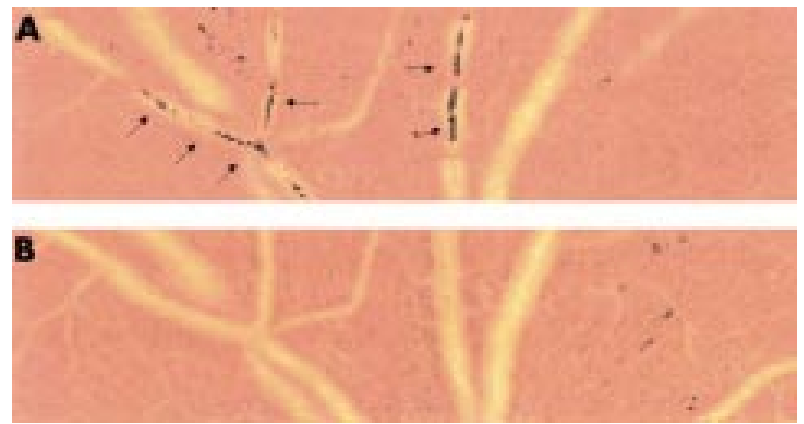

The retinal perfusion map using scanning laser Doppler flowmetry demonstrates revealed impaired perfusion of the retinal temporal artery at the 11 and 12 o'clock positions (arrows in A) with resolution within $21 \mathrm{~min}(\mathrm{~B})$

4 Ploner CJ, Valdueza JM, Villringer A. Vasospastic amaurosis fugax in a patient with overlap collagenosis treated with nimodipine. Neurol Res $1995 ; 17: 66-9$.

Josef G Heckmann, Charly Gaul, Bernhard Neundörfer Department of Neurology, University of Erlangen-Nuremberg, 91054 Erlangen, Germany

Joanna Harazny, Georg Michelson

Department of Ophthalmology, University of Erlangen-Nuremberg, 91054 Erlangen, Germany

Correspondence to: Dr Josef G. Heckmann, Department of Neurology, University of Erlangen-Nuremberg, Schwabachanlage 6, 91054 Erlangen, Germany; josef.heckmann@neuro.imed.uni.erlangen.de 